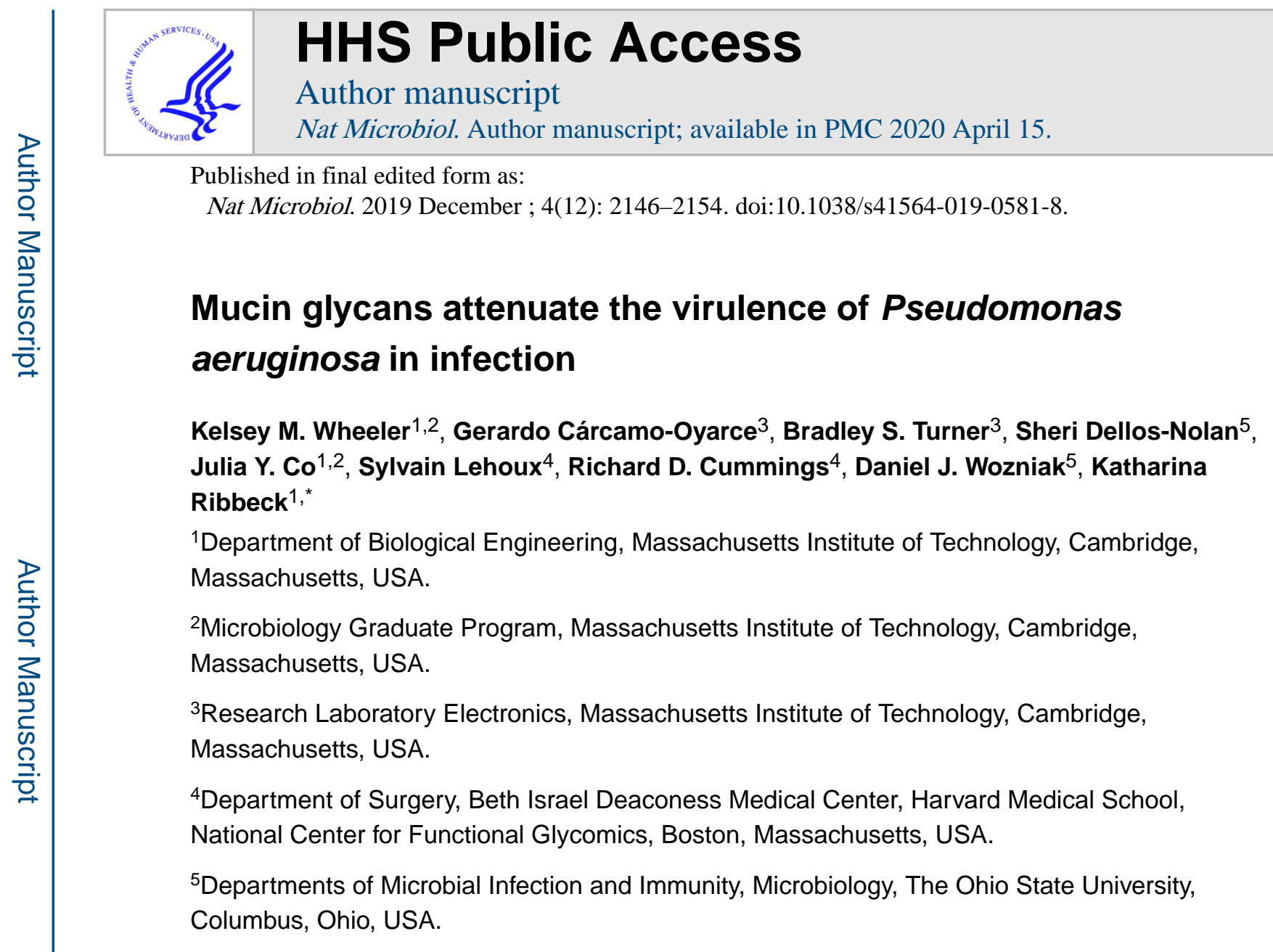

\title{
Introductory Paragraph:
}

A slimy, hydrated mucus gel lines all wet epithelia in the human body, including the eyes, lungs, gastrointestinal and urogenital tracts. Mucus forms the first line of defense while housing trillions of microbes that constitute the microbiota (1). Rarely do these microbes cause infections in healthy mucus (1), suggesting mechanisms exist in the mucus layer that regulate virulence. Using the bacterium Pseudomonas aeruginosa and a 3-dimensional (3D) laboratory model of native mucus, we determined that exposure to mucus triggers the downregulation of virulence genes involved in quorum sensing, siderophore biosynthesis, and toxin secretion, and rapidly disintegrates biofilms, a hallmark of mucosal infections. This phenotypic switch is triggered by mucins, polymers densely grafted with $O$-linked glycans that form the 3D scaffold inside mucus. Here we show isolated mucins act at various scales, suppressing distinct virulence pathways, promoting a planktonic lifestyle, reducing

\footnotetext{
Users may view, print, copy, and download text and data-mine the content in such documents, for the purposes of academic research, subject always to the full Conditions of use:http://www.nature.com/authors/editorial_policies/license.html\#terms

*Correspondence to: ribbeck@mit.edu.

Author contributions: KMW, GC-O, BST, SDN, JYC, DJW, RDC, and KR designed the experiments. KMW, GC-O, BST, SDN,

JYC, and SL conducted experiments. All authors analyzed the data and contributed to the writing of the manuscript.

Data availability

High-throughput sequencing data presented in Figures 1 and 4 are deposited in the Gene Expression Omnibus (GEO), accession number GSE136097. All other data that support the findings of this study are available from the corresponding author on reasonable request.

Competing interests: Authors declare no competing interests.
} 
cytotoxicity to human epithelia in vitro, and attenuating infection in a porcine burn model. Other viscous polymer solutions lack the same effect, indicating that mucin's regulatory function is not a result of its polymeric structure alone. We identify that interactions with $P$. aeruginosa are mediated by mucin-associated glycans. By isolating glycans from the mucin backbone, we assessed the collective activity of hundreds of complex structures in solution. Like their grafted counterparts, free mucin-glycans potently regulate bacterial phenotypes even at relatively low concentrations. This regulatory function likely depends on glycan complexity, as monosaccharides do not attenuate virulence. Thus, mucin-glycans are potent host signals that "tame" microbes, rendering them less harmful to the host.

\section{Main Text:}

To identify mechanisms in native mucus that control pathogens, we tested whether exposure to native intestinal mucus alters biofilm integrity. Our model organism was the opportunistic pathogen Pseudomonas aeruginosa PAO1, which does not trouble healthy individuals (Fig. $1 \mathrm{a})$, but can cause severe morbidity or death in people with compromised immunity, aberrant mucus production (Fig. 1b), or burn wounds $(2,3)$. Mature $P$. aeruginosa biofilms were exposed to buffer or native intestinal mucus. While buffer alone did not affect biofilm integrity (Fig. 1c), biofilms exposed to mucus dissociated from the surface and $70 \%$ of cells shifted into the planktonic phase (Fig. 1d). Since the growth rate was not altered in mucus relative to buffer alone (Fig. S1), the shift to planktonic growth may arise from phenotypic regulation by mucus. Mucus-mediated biofilm dispersal is dependent on an intact flagellum (Fig. 1c), indicating that mucin triggers an active, motility-driven escape, as opposed to mechanical disruption. To test whether mucus only affects biofilms or more broadly regulates virulence traits, we measured the expression of genes important for establishing infection (3). This revealed intestinal mucus transcriptionally suppresses quorum sensing (lasR), siderophore biosynthesis ( $p v d A$ ), and type-three secretion ( $p c r V$ ) (Fig. 1e). Native gastric and salivary mucus similarly suppressed major infection-related genes (Fig. 1e), suggesting that virulence suppression is conserved across various mucosal surfaces. Together, these findings demonstrate that mucus contains factors that modulate bacterial behaviors at the levels of gene expression and phenotype.

To identify specific regulatory factors within complex mucus, we tested mucus fractions separated according to molecular weight. The primary bioactive component of whole mucus was larger than $100 \mathrm{kDa}$ (Fig. 1f), pointing to mucin polymers as possible candidates. Mucins are a major high molecular-weight component of mucus that disperse cells in isolation (4). Adding purified mucins back into depleted mucus was sufficient to restore biofilm dispersal and virulence gene suppression (Fig. 1f-h), providing strong evidence that mucin glycopolymers constitute key regulatory cues inside mucus. Here, using natively purified mucins was critical because commercially available mucins harbor reduced chemical complexity due to the harsh purification process (5).

To directly test whether mucins trigger a global transcriptional response, we performed RNA sequencing on $P$. aeruginosa grown with or without $(0.5 \%$ w/v) MUC5AC or MUC5B, the most abundant gel-forming mucins secreted in niches colonized by $P$. aeruginosa, including the nasal and oral cavities, respiratory tract, eyes, and middle ear (Fig. 2a, b). Both mucins 
triggered a genome-wide response (Fig. 2c, Table S1) and suppressed many virulence pathways, including type 1,2,3, and 6 secretion systems, siderophore biosynthesis (pyoverdine and pyochelin), and quorum sensing (Fig. 2d, Table S2). Among the upregulated genes, we detected enrichment in the denitrification pathway (Table S3), consistent with inverse regulation by quorum sensing (6). Several metabolic genes were also differentially regulated, including those associated with fumarate metabolism and amino acid and C5-carboxylate transport (Table S1). These results are consistent with metabolic changes, which often correlate with changes to virulence (7). Importantly, we found no enrichment in virulence pathways among upregulated genes (Table S3). There were differences in the exact genes that were differentially regulated, as well as differences in the magnitudes of those changes after exposure to MUC5AC or MUC5B (Fig. 2c, Fig. S2, Table S4), suggesting differences in mucin biochemistry and specific regulatory function. We replicated this effect with various media, time points, and bacterial strains (Fig. S3, Tables S5-S7), demonstrating that mucin suppresses virulence gene expression across a range of experimental conditions.

Regulation of virulence was validated by qPCR (Fig. S4). To confirm the phenotypic relevance of these changes, we combined functional biochemical assays and infection models (Fig. 2e, i). Protease activity and siderophore production were lower in supernatants from $P$. aeruginosa exposed to mucin than in supernatants from bacteria grown in medium alone (Fig. S5). Consistent with downregulation of biofilm formation, MUC5AC suppressed P. aeruginosa PAO1 association to surfaces made of glass (Fig. S6) and plastic (Fig. S7), and reduced attachment to live HT-29 human epithelial cells in a concentration dependent manner (Fig. 2f). Mucins promoted a larger vertical distribution of $P$. aeruginosa cells than those grown in medium alone, shifting them to a suspended and less-aggregated planktoniclike state with cells still detectable $250 \mu \mathrm{m}$ from the glass surface (Fig. S6). Collectively, these data highlight two facets of mucin regulatory function: one in which mucins induce a global transcriptional response that "disarms" $P$. aeruginosa by downregulating important virulence genes, and another in which mucins suppress aggregation and bacterial attachment to surfaces. Mucin-mediated changes were sufficient to neutralize antagonistic interactions between $P$. aeruginosa and human cells. By monitoring survival of human epithelial cells over time, we determined that MUC5AC reduced $P$. aeruginosa-mediated epithelial cell death in a dose-dependent manner (Fig. 2g), while maintaining the morphology and confluency of the epithelial cell monolayer (Fig. 2h).

To better understand the relevance of mucin's regulatory function in a complex biological system of multiple cell types and an active immune system, we exposed $P$. aeruginosainfected porcine burn wounds to a wound dressing containing MUC5AC and quantified the bacterial burden over time by counting colony-forming units (CFU, Fig. 2i, S8). Exposure to MUC5AC resulted in two-log reductions in CFU in wounds 1-week post-infection, compared to no reduction in the mucin-free mock treatment (Fig. 2j). The sustained clearance of $P$. aeruginosa detected here is likely not due to direct killing by MUC5AC, as viability was not inhibited by mucins in isolation (Fig. 2k). Rather, mucin likely mediates bacterial clearance through regulation of microbial phenotypes, which attenuates pathogens and thereby facilitates host-mediated clearance. 
It is tempting to speculate that mucin triggers this phenotypic switch by maintaining cells in the planktonic, non-aggregate form. To test this hypothesis, we evaluated whether mucins still suppressed virulence genes in mutants lacking flagellar motility $(\triangle m o t A B C D)$ or the ability to aggregate ( $\triangle p s I p e I$ ) (Fig. 3a). Both mutants responded to mucins (Fig. 3b), indicating that mucin's virulence-attenuating function is not a downstream consequence of changes to motility or aggregation, but rather a parallel effect. Integrating our RNA-seq results with the major genetic components of the $P$. aeruginosa virulence network $(8,9$, Fig. $3 c)$ revealed that mucin's broad virulence-attenuating effect likely involves multiple regulatory systems. Polymer solutions like mucin have complex structural and biochemical properties that could directly or indirectly trigger signaling events through many sensory systems in $P$. aeruginosa. One possibility is that mucins may trigger a general response through their electrostatic or hydrophobic properties or by creating geometric constraints. An alternative hypothesis is that the observed response is triggered by sensing specific biochemical moieties that are presented on secreted mucins. To distinguish between these two possibilities, we tested whether carboxymethylcellulose (CMC), a well-established mucin mimetic with charge and viscoelastic properties similar to those of native mucins (10), could elicit changes in $P$. aeruginosa virulence. Importantly, exposure to CMC did not differentially regulate signature virulence genes (Fig. 3d), prevent surface attachment (Fig. S9), or protect epithelial cells from $P$. aeruginosa-induced death (Fig. S9). These results suggest that mucin's polymeric structure is not sufficient to mediate these virulenceattenuating effects. Rather, specific biochemistry present in mucins, but not CMC, is necessary to attenuate $P$. aeruginosa virulence.

Native mucins display a plethora of complex glycan structures covalently linked to serine/ threonine (11-13), creating a wealth of biochemical information with the potential to influence microbial gene expression. $P$. aeruginosa employs many strategies to sense and respond to host signals, allowing it to coordinate the switch between its pathogenic and hostcompatible states $(8,9)$. Sensing signature mucin-glycan motifs may be an effective mechanism to limit production of metabolically costly virulence factors in niches where virulence would not be advantageous. The potential of complex mucin-glycans to regulate microbial behavior has been largely overlooked owing to fundamental technical limitations, including the difficulty of purifying intact mucins, non-standard methods for isolating $O$ linked glycans, and the analytical complexity of predicting glycan structures (14). To determine whether glycans contribute to mucin's virulence-neutralizing capability, we assessed the degree to which glycans isolated from the MUC5AC protein backbone recapitulate the mucin response in $P$. aeruginosa. We isolated mucin-glycans via alkaline $\beta$ elimination, which preserved the unique sequences in the oligosaccharide chains and yielded a pooled library of $O$-linked glycans (Fig. 4a, b). We then analyzed glycans via matrixassisted laser desorption/ionization time-of-flight (MALDI-TOF). After processing for annotation and assignment based on mass and known core structures, we identified $>90$ glycan structures (Fig. 4c, Table S8), not including distinct isomeric forms, which likely would increase the library diversity to several hundred structures. The diversity of our glycan library is comparable to those of published libraries for mammalian gastric and respiratory mucins $(11-13,15)$. Specifically, glycans in our library were predominantly built on core-2 
[Gal $\beta 1-3($ GlcNAc$\beta 1-6)$ GalNAca 1-] and, to a lesser extent, core-1 (Gal $\beta 1-3$ GalNAca 1-) structures, with a high level of fucosylation and relatively little sialylation $(11-13,15)$.

Like native mucus and intact mucins, mucin-glycans triggered an active, motility-dependent dispersal of bacterial cells from mature biofilms (Fig. 4d, S10) into the planktonic state (Fig. 4e). Mucin-glycans also prevented bacterial attachment to glass surfaces (Fig. S11) and to human cells (Fig. 4f) and yielded bacterial aggregates smaller than those formed in medium alone or with monosaccharides (Fig. 4g). To determine which mucin-regulated pathways were specifically altered in response to mucin-glycans, we exposed $P$. aeruginosa to a pool of potential glycan cues and monitored changes in gene expression. RNA-seq revealed that $P$. aeruginosa exposed to relatively low concentrations $(0.01 \% \mathrm{w} / \mathrm{v})$ of free MUC5ACglycans undergo a transcriptional response that mirrors that associated with exposure to $0.5 \%$ (w/v) whole native mucin comprised of up to $0.4 \%$ (w/v) grafted-glycans (Fig. 4h). Functional enrichment analyses confirmed that the same virulence pathways suppressed by intact MUC5AC were also suppressed by MUC5AC-glycans (Fig. 4i), further indicating that an integral part of mucin rather than another mucus-associated factor is the primary virulence-neutralizing agent in mucus. We anticipate that increasing the glycan concentration to levels present on mucin will improve the dynamic range of the transcriptional response. Importantly, $P$. aeruginosa viability was not altered by the presence of monosaccharides represented in mucin-glycans (Fig. $4 \mathrm{j}$ ). Exposure of $P$. aeruginosa to a pool of these monosaccharides did not trigger dispersal (Fig. 4d), prevent attachment to glass (Fig. S11) or human cells (Fig. 4e), or differentially regulate quorum sensing (lasR), siderophore production ( $p v d A$ ), or type-three secretion ( $p c r V$ ) genes (Fig. $4 \mathrm{k}$ ). Monosaccharide exposure also did not suppress virulence-factor production, even with increasing concentrations (Fig. S12), although a non-significant effect on bacterial burden in vivo was detected (Fig. 41). Based on these data, we conclude that the complex arrangement and particular stereochemistry of these sugar residues is critical to their function as regulatory signals.

The diversity of $O$-glycans on mucins exceeds even that on tissue surfaces (16-18), and their complexity makes them ideal for encoding biological information with a high degree of specificity. In this way, mucins present and retain a myriad potential regulatory cues. We propose that microbes likely evolved mechanisms to recognize, process, uptake, and respond to specific moieties within the complex array of mucin-glycans (19). The question remains how mucin-glycans interact with, and are sensed by, $P$. aeruginosa at a molecular level. One possibility is that glycans may directly serve as signal through a carbohydrate binding site in a global regulatory system, such as those affecting the secondary messenger c-di-GMP or the non-coding RNAs $r s m Y$ and $r s m Z(8,9)$. Potential glycan sensors have been identified in $P$. aeruginosa that are thought to feed into regulatory virulence pathways and have annotated carbohydrate binding sites, such as the two-component sensor LadS and the diguanyulate cyclase NicD $(20,21)$. Mucin-glycans may also trigger metabolic changes by serving as a nutritional substrate or regulate signaling pathways through interactions with specific $P$. aeruginosa lectins or surface adhesins. We speculate that the structural diversity of mucins and mucin-associated glycans permits them to interact with several bacterial receptors and mediate distinct functions. 
Collectively, our findings reveal a previously unrecognized role for mucin-glycans as potent host-derived regulators of bacterial phenotype, which has broad implications for how the body prevents mucosal infections while maintaining a diverse microbiota. Why diseased mucus no longer retains the ability to attenuate virulence (22-26) remains an open question. Based on our findings that glycans trigger a switch in bacterial phenotype, we postulate that changes to mucin glycosylation patterns in disease, such as increased sialylation (15), will alter both mucin's binding properties with microbes and its protective function. By isolating mucin-glycan library, we have established the conceptual and technical framework to systematically address these open questions about the glycan regulatory code. We posit that cracking this code will elucidate the influence of glycans on the virulence of microbes that interact with the host via mucosal surfaces. Identification of specific bioactive glycans will likely reveal a class of therapeutics for treating intractable bacterial infections and may inspire treatment strategies that tune host glycan signals to attenuate virulence and stabilize the healthy microbiota.

\section{Materials and Methods}

\section{Strains and growth conditions.}

Batch cultivation of Pseudomonas aeruginosa strains (Table S9) was carried out shaking at $37{ }^{\circ} \mathrm{C}$ in LB (Difco). Thirty $\mu \mathrm{g} / \mathrm{mL}$ gentamicin was added to the medium for strains with gentamicin-resistant plasmids.

For whole-mucus experiments, overnight cultures of PAO1 were diluted 10-fold into ABTGC (ABT minimal medium (27) supplemented with $5 \mathrm{~g}$ glucose and $5 \mathrm{~g}$ casamino acids) and grown for $4 \mathrm{~h}$. Seventy-five microliters of these cultures were exposed to $75 \mu \mathrm{L}$ of solubilized mucus or solubilization buffer (described in "preparation of whole mucus") for 1 $\mathrm{h}$ at $37^{\circ} \mathrm{C}$ in a static 96 -well microtiter plate (Cellstar).

For experiments involving liquid culture, overnight cultures of PAO1 were diluted to an $\mathrm{OD}_{600}$ of 0.01 into $150 \mu \mathrm{L}$ ABTGC with or without $(0.01-0.5 \%)$ MUC5AC, $(0.5 \%)$ MUC5B, $0.5 \%$ carboxymethylcellulose (CMC, Sigma), (0.01\%) MUC5AC-glycans, or $(0.01 \%)$ monosaccharide mixture and grown for $5 \mathrm{~h}$ at $37{ }^{\circ} \mathrm{C}$ in a static 96 -well microtiter plate. Note that based on the data in Figure 4B, the monosaccharide mixture contained equal weights of galactose, $\mathrm{N}$-acetylglucosamine [GlcNAc], N-acetylgalactosamine [GalNAc], fucose, and sialic acid (all sugars purchased from Sigma). The concentration of glycans was selected based on the mucin dose response curves in Figure 2. In these experiments, we found that $0.01 \%$ was the lowest mucin concentration with measurable effects (Fig. 2F). Due to the technical challenges of preparative-scale purification of mucin-glycans, we used the minimal inhibitory concentration in this work.

\section{Human saliva collection.}

Submandibular saliva was collected from human volunteers using a custom vacuum pump, pooled, centrifuged at $2500 \mathrm{x}$ g for 5 minutes, and protease inhibitors were added as previously described (28). Human saliva samples were collected after explaining the nature and possible consequences of the studies, obtaining informed consent, and receiving 
approval from the institutional review board and Massachusetts Institute of Technology's Committee on the Use of Humans as Experimental Subjects under protocol \#1312006096.

Preparation of whole mucus.

Mucus was scraped from fresh pig stomachs and intestines, and solubilized (1 $\mathrm{g}$ scrapings to $5 \mathrm{~mL}$ ) in $0.2 \mathrm{M}$ sodium chloride buffer with protease inhibitors ( $5 \mathrm{mM}$ Benzamidine $\mathrm{HCl}, 1$ $\mathrm{mM}$ dibromoacetophenone, $1 \mathrm{mM}$ phenylmethylsulfonylfluoride (PMSF), and 5mM EDTA at $\mathrm{pH} 7$ ) and $0.04 \%$ sodium azide (Sigma). Cellular debris and food waste was removed via low-speed centrifugation, $8000 \mathrm{x} \mathrm{g},\left(7,000 \mathrm{rpm}\right.$ Sorvall GS-3 rotor), for 30 minutes at $4{ }^{\circ} \mathrm{C}$.

\section{Mucin purification.}

This study used native porcine gastric mucins (MUC5AC), porcine intestinal mucins (MUC2), and human salivary mucins (MUC5B), which differ from industrially purified mucins in their rheological properties and bioactivity $(4,5,28-30)$. Native mucins were purified as previously reported $(4,28-31)$. Briefly, mucus was scraped from fresh pig stomachs and intestines, solubilized in sodium chloride buffer (described above), and insoluble material was removed by ultracentrifugation at 190,000 x g RCF for $1 \mathrm{hr}$ at $4{ }^{\circ} \mathrm{C}$ (40,000 rpm, Beckman 50.2 Ti rotor with polycarbonate bottles). Submandibular saliva was collected from human volunteers using a custom vacuum pump, pooled, centrifuged, and protease inhibitors were added (28). Mucins were purified using size-exclusion chromatography on separate Sepharose CL-2B columns. Mucin fractions were then desalted, concentrated, and lyophilized for storage at $-80^{\circ} \mathrm{C}$. Lyophilized mucins were reconstituted by shaking them gently at $4{ }^{\circ} \mathrm{C}$ overnight in the desired medium. Mass spectrometry is routinely used to monitor the composition of purified mucin extracts $(30,31)$. This type of analysis has shown that mucin extracts purified from porcine stomach mucus, for example, are composed predominantly of MUC5AC, with small quantities of MUC2, MUC5B, and MUC6, as well as histones, actin, and albumin $(30,31)$.

\section{Isolation of mucin oligosaccharide.}

This study applied non-reductive alkaline $\beta$-elimination ammoniolysis to dissociate nonreduced glycans from mucins (32). Purified mucins were dissolved in ammonium hydroxide saturated with ammonium carbonate and incubated at $60{ }^{\circ} \mathrm{C}$ for $40 \mathrm{~h}$ to release oligosaccharide glycosylamines and partially deglycosylated mucins. Volatile salts were removed via repeated centrifugal evaporation and the oligosaccharide glycosylamines were separated from residual deglycosylated mucins via centrifugal filtration through $3-5 \mathrm{kDa}$ molecular weight cut-off membranes in accordance with the manufacturer's instructions (Amicon Ultracel). The resulting oligosaccharide glycosylamines were converted to reducing oligosaccharide hemiacetals via treatment with boric acid. Residual boric acid was removed via repeated centrifugal evaporation from methanol. Oligosaccharides were further purified via solid-phase extraction through Hypercarb mini-columns (ThermoFisher) and residual solvents were removed through centrifugal evaporation (33). 


\section{Capillary electrophoresis of oligosaccharides.}

Reducing oligosaccharides released from mucins were labeled via reductive amination with the fluorescent tag 8-aminopyrene-1,3,6-trisulfonic acid, sodium cyanoborohydride, and citric acid. Labeled oligosaccharides were analyzed with polyvinylalcohol coated N-CHO capillaries in accordance with the manufacturer's (SciEx/Beckman) protocol using a PA800 (Beckman) capillary electrophoresis instrument, detected with laser-induced fluorescence, and analyzed with 32Karat software. The relative sizes of separated oligosaccharides were determined by comparison with migration times of glucose polymer standards.

Chromatograms were constructed in GraphPad Prism (v7.04).

\section{Capillary electrophoresis of monosaccharides.}

The monosaccharide composition of released mucin glycans was determined through established methods $(34,35)$. Briefly, neutral monosaccharides were obtained by hydrolysis in trifluoracetic acid for $1 \mathrm{~h}$ at $80^{\circ} \mathrm{C}$. The liberated monosaccharides were labeled with 8aminopyrene-1,3,6-trisulfonic acid via reductive amination as described for oligosaccharides. Monosaccharides were analyzed with capillary electrophoresis on a bare silica column and detected via laser-induced fluorescence. Monosaccharides were identified and quantified by comparison to a maltose internal standard and migrations times and standard curves were generated using purified standard sugars. Chromatograms were constructed in GraphPad Prism (v7.04).

\section{Matrix-assisted laser desorption/ionization time-of-flight (MALDI-TOF) and prediction of mucin-glycan structure.}

$\beta$-eliminated glycans were permethylated and analyzed at the Glycomics Core at Beth Israel Deaconess Medical Center as previously described (36). Mass spectrometry data were acquired on an UltraFlex II MALDI-TOF Mass Spectrometer (Bruker Daltonics). Reflective positive mode was used, and data were recorded between $500 \mathrm{~m} / z$ and $6000 \mathrm{~m} / z$. The mass spectrometry $O$-glycan profile was acquired by aggregating at least 20,000 laser shots. Mass peaks were manually annotated and assigned to a particular $O$-glycan composition based on known core structures.

\section{RNA preparation.}

Total RNA was extracted using the MasterPure RNA Purification kit (Lucigen) and residual DNA was removed using the Turbo DNA-free kit (Ambion). The integrity of the total RNA was assessed with an Agilent 2100 Bioanalyzer (Agilent Technologies). 16S, 23S, and 5S rRNA were removed using the Ribo-Zero Magnetic Kit (Bacteria) (Epicentre).

\section{RNA sequencing.}

Gene expression analysis was conducted via Illumina RNA sequencing (RNA-seq). RNAseq was conducted for three biological replicates. Libraries were produced using the KAPA RNA HyperPrep kit (Kapa Biosystems). Libraries were sequenced using the Illumina HiSeq platform with a single-end protocol and read lengths of $40 \mathrm{nt}$ or $50 \mathrm{nt}$. 


\section{Analysis of sequencing data.}

Sequence reads were mapped onto the $P$. aeruginosa PAO1 reference genome, which is available for download from the Pseudomonas Genome Database (http:// www.pseudomonas.com) using the Galaxy server (37). Gene expression values were normalized based on library size and differentially expressed genes were identified using a negative binomial test with a false discovery rate (FDR) less than 0.05. Expression changes in signature virulence genes identified by RNA-seq were validated with quantitative PCR (qPCR), which revealed strong concordance between the two methods (Fig. S4).

Functional category (pathway) assignments were downloaded from the Pseudomonas Genome Database. Pathway enrichment analysis was performed in MATLAB (R2016b) using the one-sided Mann-Whitney U test, where ranking was based on the log2transformed fold change.

Heatmaps and scatter plots of gene expression data were constructed in GraphPad Prism. Principle component analysis was performed in R (3.4.0) using the DESeq 2 workflow (38). For all analyses of sequencing data, $p$-values were adjusted for multiple comparisons with the Benjamini-Hochberg correction to obtain the FDR.

\section{qRT-PCR analysis.}

Primers used in this study are listed in Table S9. Quantitative reverse transcriptase PCR (qRT-PCR) was performed using a two-step method. First-strand cDNA was synthesized from total RNA using the ProtoScript II First Strand cDNA Synthesis kit (NEB). The cDNA was used as a template for qRT-PCR with a SYBR PowerUp Master Mix kit (Applied Biosystems by Life Technologies) on a Roche LightCycler 480 Real-Time PCR System. Primers for qRT-PCR were designed based on previously published literature or using the NCBI Primer-BLAST tool (https://www.ncbi.nlm.nih.gov/tools/primer-blast/). The genes $r p o D$ and proC were used as endogenous controls. The elimination of contaminating DNA was confirmed via qPCR amplification of $r p o D$ on control samples that did not have reverse transcriptase added during cDNA synthesis. Melting-curve analyses were employed to verify single-product amplification. Change in gene expression was calculated based on mean change in qPCR cycle threshold (dCt) using the $\Delta \Delta \mathrm{Ct}$ method (Fold change $=2^{-\Delta \Delta \mathrm{Ct}}$ ).

\section{Dispersal of static $P$. aeruginosa biofilms.}

Biofilm dispersal under static conditions P. aeruginosa biofilm dispersal was assayed as previously described (4), with slight modification. Briefly, an overnight culture of PAO1GFP or PAO1-GFP $\triangle f l i D$ was diluted in ABTGC medium to an initial OD600 of 0.01, added to a glass-bottom or plastic 96-well plate, and incubated for $48 \mathrm{~h}$ at $37^{\circ} \mathrm{C}$ under static conditions. The supernatant containing non-adherent cells was removed from the plate and the biofilm remaining in each well was washed at least three times with $0.9 \% \mathrm{NaCl}$. Biofilms were exposed to whole mucus, the solubilization buffer, ABTGC medium alone, or ABTGC medium $+0.01 \%$ mucin-glycans or monosaccharides. Biofilms were statically incubated at $37{ }^{\circ} \mathrm{C}$ for $3 \mathrm{~h}$. Plates were washed three times with $0.9 \% \mathrm{NaCl}$ and resuspended in ABTGC medium, then examined via microscopy to determine the remaining biofilm biomass. Viable dispersed cells were quantified via CFU counts on LB agar plates. Image 
acquisition was performed using a confocal laser scanning microscope (LSM 800; Zeiss) equipped with a $63 \times / 1.4$ NA oil immersion or a $100 \times / 1.4$ NA oil immersion objective. The excitation wavelength for GFP was $488 \mathrm{~nm}$. At least five stacks were recorded for each well and at least three independent wells. Biofilm quantification was performed using COMSTAT (1.66).

\section{Measurement of protease activity.}

Bacterial cultures were pelleted via centrifugation $(13,200 \times g$ for $3 \mathrm{~min}$ at room temperature), and supernatants were filtered-sterilized using a $0.2 \mu \mathrm{m}$ filter.

\section{Protease IV activity.}

The activity of protease IV in cell-free culture supernatants was measured via breakdown of the chromogenic substrate Chromozym PL (Roche), which reacts specifically with protease IV in $P$. aeruginosa culture supernatants (39). Briefly, $10 \mu \mathrm{L}$ pre-treated sample and $3 \mu \mathrm{L}$ Chromozym PL (7 mM) were combined in reaction buffer $(20 \mathrm{mM}$ Tris/HCl, $\mathrm{pH}$ 8.0) to a total volume of $100 \mu \mathrm{L}$ in a microwell plate. Plates were assayed on a SpectraMax M Series Multi-Mode Microplate Reader (Molecular Devices) pre-equilibrated to $30^{\circ} \mathrm{C}$ by measuring the rate of increase in absorbance at $405 \mathrm{~nm}$ at 3-min intervals for $30 \mathrm{~min}$. Protease IV activity was calculated using the following equation:

$$
F \times E \times d \times \Delta \mathrm{A} / \mathrm{min}
$$

where $F$ is the dilution factor, $E$ is the extinction coefficient (at $405 \mathrm{~nm}=10.4$ ), $d$ is the path length (at $100 \mu \mathrm{L}$ volume in a microtiter plate $\approx 0.53 \mathrm{~cm}$ ), and $\Delta \mathrm{A} / \mathrm{min}$ is the maximum change in absorbance per min. The protease activity was normalized to account for variation in bacterial cell growth based on the optical density (absorbance at $600 \mathrm{~nm}$ ) of the culture at $5 \mathrm{~h}$. Relative changes were calculated based on the protease activity in medium alone.

\section{Alkaline protease activity.}

Alkaline protease activity was tested using a modified method previously described (40). Samples containing $1 \mathrm{mg}$ of Hide powder azure (Sigma) dissolved in buffer $(0.075 \mathrm{~mL})$ consisting of $20 \mathrm{mM}$ Tris/ $\mathrm{HCl}$, $\mathrm{pH} 8.0$ and $1 \mathrm{mM} \mathrm{CaCl}_{2}$ were mixed with $0.025 \mathrm{~mL}$ of the culture supernatants. The reaction mixtures $(0.1 \mathrm{~mL})$ were incubated at $37^{\circ} \mathrm{C}$ for $1 \mathrm{~h}$. Undissolved substrate was removed via centrifugation at $4000 \times g$ for $5 \mathrm{~min}$. The absorbance of the reaction mixtures was determined at $595 \mathrm{~nm}$ on a SpectraMax M Series Multi-Mode Microplate Reader (Molecular Devices). Protease activity was expressed in terms of protease units per milliliter $(\mathrm{U} / \mathrm{mL})$, where one unit is equivalent to an increase in $\mathrm{OD}_{595}$ of 1.0 per hour at $37^{\circ} \mathrm{C}$. Protease activity was normalized to account for variation in bacterial cell growth based on the $\mathrm{OD}_{600}$ of the culture at $5 \mathrm{~h}$. Relative changes were calculated based on the protease activity in medium alone.

\section{Measurement of siderophore fluorescence.}

Pyoverdine and pyochelin levels were simultaneously quantified based on characteristic fluorescence spectra, as previously described (41). Briefly, fluorescence was measured on a 
SpectraMax M Series Multi-Mode Microplate Reader (Molecular Devices). Pyoverdine production was quantified using an excitation wavelength of $400 \mathrm{~nm}$ and an emission wavelength of $460 \mathrm{~nm}$. Pyochelin fluorescence was quantified using an excitation wavelength of $350 \mathrm{~nm}$ and an emission wavelength of $410 \mathrm{~nm}$. To account for the background fluorescence of pyoverdine, pyochelin production was calculated using the equation:

$$
z=w-3 \mathrm{E}-7 \times y^{2}-0.0413 \times y
$$

where $z$ is the actual value of pyochelin production, $W$ is the fluorescence measured at excitation/emission 350/410, and $y$ is the pyoverdine fluorescence measured at excitation/ emission 400/460.

\section{Quantification of bacterial growth and polystyrene-attached biomass.}

Growth curves were measured in microtiter plates on a SpectraMax M Series Multi-Mode Microplate Reader (Molecular Devices) by measuring the absorbance at $600 \mathrm{~nm}$ and by CFU counts. Adherent biomass was quantified using crystal violet (42). Cells attached to the wells of the plate were washed three times with $0.9 \% \mathrm{NaCl}$ and stained with $0.01 \%$ crystal violet for $15 \mathrm{~min}$ at room temperature. Wells were washed three times with $0.9 \% \mathrm{NaCl}$. Ethanol was added to each well and the absorbance at $595 \mathrm{~nm}$ was measured after $15 \mathrm{~min}$. The absorbance in each well was normalized based on the optical density of the culture at 5 h.

\section{Human cell culture.}

Authenticated, mycoplasma-free HT-29 cells (ATCC® HTB38 ${ }^{\mathrm{TM}}$ ), a human carcinoma cell line with epithelial morphology were obtained directly from the American Type Culture Collection (ATCC). Morphology was routinely monitored to verify cell line authenticity and cells were periodically tested for contamination by PCR. Cells were cultured in Dulbecco's Modified Eagle Medium GlutaMAX (DMEM) (Gibco) supplemented with 10\% fetal bovine serum (FBS) (Gibco) at $37{ }^{\circ} \mathrm{C}$ in $5 \% \mathrm{CO}_{2}-95 \%$ air. Cells were split 1:12 and passaged as the culture reached confluence. To prepare for co-culture with bacteria, HT-29 cells were detached from the growth surface with $0.25 \%$ trypsin-1 mM EDTA (Gibco), resuspended in DMEM+FBS, counted, diluted to the appropriate density in DMEM+FBS, and seeded in 96well plates to confluence (approximately $5 \times 10^{5} \mathrm{HT}-29$ cells).

\section{Examination of bacterial interactions with epithelial cells.}

In all experiments, control and experimental HT-29 cells were identically treated except that co-culture medium did not contain bacteria in the uninfected controls. Bacteria were cocultured with HT-29 cell monolayers at an initial multiplicity of infection (MOI) of 20 (1 $\times$ $10^{7} \mathrm{CFU}$ ) for 1-8 h. Subsequently, HT-29 cells were processed and assayed in the cellfunction assays described below.

Analysis of bacterial attachment.-After $1 \mathrm{~h}$ of co-culture, non-adherent bacteria in the supernatant were aspirated and quantified by serial dilution as CFUs. HT-29 cell 
monolayers were washed three times with phosphate-buffered saline (PBS) (Gibco), and cells were lysed with 1\% Triton X-100 (Sigma) and removed from the growth surface. Attached bacteria were serially diluted in PBS and quantified as CFUs.

Epithelial cytotoxicity assay.-HT-29 cell killing by $P$. aeruginosa was measured using the membrane impermeable nuclear stain propidium iodide (PI), which allows continuous quantitative measurement of cell viability over time, owing to its optimal linear DNAbinding, without background from bacterial cell death (Fig. S14). After co-culture of 1-10 h, PI fluorescence (excitation/emission 535/617 nm) was measured on a SpectraMax M Series Multi-Mode Microplate Reader (Molecular Devices). Between measurements, plates were kept in an incubator at $37^{\circ} \mathrm{C}, 5 \% \mathrm{CO}_{2}-95 \%$ air. At the end of the experiment, maximal fluorescence was measured following treatment of each well with $1 \%$ Triton X-100 to permeabilize all cells and label all nuclei with PI, which corresponds to $100 \%$ cell death. Background fluorescence of PI was measured in uninfected control cells (exposed to medium alone or the appropriate concentration of MUC5AC) at the beginning of the experiment. Percentage cytotoxicity was calculated as:

$$
\left(f-f_{0}\right) /\left(M-f_{0}\right) \times 100
$$

where $f_{O}$ is the initial fluorescence, $M$ is the maximum fluorescence after addition of Triton $\mathrm{X}-100$, and $f$ is the fluorescence at any given time. Mucin dose response curves were assessed at $7.5 \mathrm{~h}$.

\section{Confocal imaging.}

Image acquisition was performed using a confocal laser scanning microscope (LSM 800; Zeiss) equipped with a $63 \times / 1.4$ NA oil immersion or a $100 \times / 1.4$ NA oil immersion objective. Images were analyzed with Zeiss ZEN 2.1 imaging software. The excitation wavelengths for GFP and PI were $488 \mathrm{~nm}$ and $535 \mathrm{~nm}$, respectively. 3D images of attached and non-attached cells were created with IMARIS 7.7.2. Plots were generated in GraphPad Prism. Quantification of bacterial aggregate volume was performed using COMSTAT (1.66).

\section{Examination of bacterial interactions with porcine burn wounds.}

Interactions between $P$. aeruginosa and full-thickness burn wounds were assessed as previously described (43). Briefly, Female Yorkshire pigs $(n=4)$ weighing between 70 and 80 pounds were anesthetized and the dorsal trunk was shaved and surgically prepared. Under aseptic conditions, six 2"x2" inch full-thickness burn wounds were created on the back of the pig using an electrically heated burn device with controlled pressure delivery for $50 \mathrm{~s}$. One day after the thermal injury, mid-log phase cultures of $P$. aeruginosa were topically inoculated onto the wound site at a concentration of $1 \times 10^{5} \mathrm{CFU}$ in $250 \mu \mathrm{L}$ of $20 \% \mathrm{PF}-127$ (Sigma) prepared in phosphate-buffered saline with $0,0.05 \%(0.5 \mathrm{mg} / \mathrm{mL})$, or $0.5 \%(5.0$ $\mathrm{mg} / \mathrm{mL}$ ) MUC5AC or $0.01 \%(0.1 \mathrm{mg} / \mathrm{mL})$ MUC5AC-glycans or monosaccharides. The inoculated and treated wound was rubbed with a sterile spatula for $30 \mathrm{~s}$. On days 1 and 4 after inoculation, treatment was reapplied to the wound. On days 1 and 7 after inoculation, full-thickness wound-tissue biopsies were collected for microbiological analysis using a 6- 
$\mathrm{mm}$ sterile disposable punch biopsy tool. Treatments for each wound site were randomized, and not blinded.

Viable bacterial counts were determined from three randomly selected 6-mm punch biopsies from each wound site. Biopsies were weighed and placed in separate sterile polypropylene culture test tubes containing $1 \mathrm{~mL}$ of PBS. All samples were homogenized with a Pro Scientific Bio-Gen Series Pro200 hand-held homogenizer for 45 s. The resulting solutions were serially diluted and plated on Pseudomonas Isolation Agar PIA-rifampicin (100 $\mu \mathrm{g} / \mathrm{mL}$ ) in at least triplicate and incubated at $37{ }^{\circ} \mathrm{C}$ overnight. CFU were calculated per gram of tissue.

Sample size calculation.-Statistical experts in the OSU Center for Biostatistics have performed sample size calculations based on power analysis. To determine minimum sample sizes necessary to detect some of the effects under investigation, data from previous preliminary studies was used. While power analysis (based on $a=0.05$, two-tail tests) indicated 6 animals per group would be required to achieve statistical significance, 1-2 animals per group showed significant effects. Since the data are not normally distributed, non-parametric Kruskal-Wallis one-way analysis of variance was used.

All care of laboratory animals was in accordance with institutional guidelines, and approved by the Ohio State University Institutional Laboratory Animal Care and Use Committee (ILACUC) under protocol 2008A0012-R1.

\title{
Statistical analysis.
}

Unless noted otherwise, experiments were done with at least three biological replicates consisting of at least three technical replicates and results are presented as mean \pm standard error. Statistical significance was assessed by an ordinary one-way ANOVA, followed by Dunnett's multiple comparisons test, or a one-sample Student's $t$ test for normalized data unless otherwise noted. Adjusted $p$-values $<0.05$ were considered significant.

\section{Supplementary Material}

Refer to Web version on PubMed Central for supplementary material.

\section{Acknowledgments:}

We thank Steven Lory for helpful comments. We thank Life Science Editors for editing assistance.

\begin{abstract}
Funding: This work was supported by NIBIB/NIH Grant R01 EB017755-04 (OSP 6940725), the National Science Foundation Career award PHY-1454673, funding from the Deshpande Center for Technological Innovation, and the MRSEC Program of the National Science Foundation under award DMR-1419807 (to KR), NIH Grant P41GM103694 (to RDC), and NIEHS/NIH Grant P30-ES002109. DJW is supported by NIH grants R01AI34895 and R01AI097511. This material is based upon work supported by the National Science Foundation Graduate Research Fellowship under Grant No. 1745302 and the MIT/NIGMS Biotechnology Training Program grant 5T32GM008334-28 (to KMW). GC-O is supported by the Early Postdoc Mobility Fellowship of the Swiss National Science Foundation (grant P2ZHP3_164844).
\end{abstract}




\section{References:}

1. Schroeder BO. Fight them or feed them: how the intestinal mucus layer manages the gut microbiota. Gastroenterol Rep (Oxf). 7(1):3-12 (2019). doi:10.1093/gastro/goy052 [PubMed: 30792861]

2. Sadikot RT, Blackwell TS, Christman JW, Prince AS. Pathogen-host interactions in Pseudomonas aeruginosa pneumonia. Am. J. Respir. Crit. Care Med 171, 1209-1223 (2005). [PubMed: 15695491]

3. Parsek MR, Singh PK. Bacterial biofilms: An emerging link to disease pathogenesis. Annu. Rev. Microbiol 57, 677-701 (2003). [PubMed: 14527295]

4. Co JY, Cárcamo-Oyarce G, Billings N, Wheeler KM, Grindy SC, Holten-Andersen N, Ribbeck K. Mucins trigger dispersal of Pseudomonas aeruginosa biofilms. npj Biofilms Microbiomes (2018). doi:10.1038/s41522-018-0067-0C.

5. Wagner E, Wheeler KM, Ribbeck K. Mucins and their role in shaping the functions of mucus barriers. Annu. Rev. Cell Dev. Biol (2018).

6. Toyofuku M, Nomura N, Fujii T, Takaya N, Maseda H, Sawada I, Nakajima T, Uchiyama H. Quorum sensing regulates denitrification in Pseudomonas aeruginosa PAO1. J. Bacteriol 189, 49694972 (2007). [PubMed: 17449629]

7. Rohmer L, Hocquet D, Miller SI. Are pathogenic bacteria just looking for food? Metabolism and microbial pathogenesis. Trends in Microbiology. (2011).

8. Jimenez PN, Koch G, Thompson JA, Xavier KB, Cool RH, Quax WJ. The multiple signaling systems regulating virulence in Pseudomonas aeruginosa. Microbiol. Mol. Biol. Rev 76 (1), 46-65 (2012). doi:10.1128/MMBR.05007-11 [PubMed: 22390972]

9. Balasubramanian D, Schneper L, Kumari H, Mathee K. A dynamic and intricate regulatory network determines Pseudomonas aeruginosa virulence. Nucleic Acids Res. 41, 1-20 (2013). [PubMed: 23143271]

10. Van Der Reijden WA, Veerman ECI, van Nieuw Amerongen A. Rheological properties of commercially available polysaccharides with potential use in saliva substitutes. Biorheology. 31, 631-42 (1994). [PubMed: 7696637]

11. Jin C, Kenny DT, Skoog EC, Padra M, Adamczyk B, Vitizeva V, Thorell A, Venkatakrishnan V, Lindén SK, Karlsson NG. Structural diversity of human gastric mucin glycans. Mol. Cell. Proteomics 16, 743-758 (2017). [PubMed: 28461410]

12. Karlsson NG, Nordman H, Karlsson H, Carlstedt I, Hansson GC. Glycosylation differences between pig gastric mucin populations: a comparative study of the neutral oligosaccharides using mass spectrometry. Biochem. J 326, 911-917 (1997). [PubMed: 9307045]

13. Holmen Larsson JM, Thomsson KA, Rodriguez-Pineiro AM, Karlsson H, Hansson GC. Studies of mucus in mouse stomach, small intestine, and colon. III. Gastrointestinal Muc5ac and Muc2 mucin O-glycan patterns reveal a regiospecific distribution. AJP Gastrointest. Liver Physiol 305, G357G363 (2013).

14. Cummings RD, Pierce JM. The challenge and promise of glycomics. Chem. Biol 21, 1-15 (2014). [PubMed: 24439204]

15. Xia B, Royall JA, Damera G, Sachdev GP, Cummings RD. Altered O-glycosylation and sulfation of airway mucins associated with cystic fibrosis. Glycobiology. 15, 747-775 (2005). [PubMed: 15994837]

16. Nakano M, Saldanha R, Göbel A, Kavallaris M, Packer NH. Identification of glycan structure alterations on cell membrane proteins in Desoxyepothilone B resistant leukemia cells. Mol. Cell. Proteomics 10, M111.009001 (2011).

17. Lee SH, Yu SY, Nakayama J, Khoo KH, Stone EL, Fukuda MN, Marth JD, Fukuda M. Core2 Oglycan structure is essential for the cell surface expression of sucrase isomaltase and dipeptidyl peptidase-IV during intestinal cell differentiation. J. Biol. Chem 285, 37683-37692 (2010). [PubMed: 20841351]

18. Yamada K, Kinoshita M. Comparative studies on the structural features of O-glycans between leukemia and epithelial cell lines. Proteome Res. 8, 521-537 (2009).

19. Varki A. Biological roles of glycans. Glycobiology. 27, 3-49 (2017). [PubMed: 27558841] 
20. Ventre I, Goodman AL, Vallet-Gely I, Vasseur P, Soscia C, Molin S, Bleves S, Lazdunski A, Lory S, Filloux A. Multiple sensors control reciprocal expression of Pseudomonas aeruginosa regulatory RNA and virulence genes. Proc. Natl. Acad. Sci (2006).doi: 10.1073/pnas.0507407103

21. Basu Roy A, Sauer K. Diguanylate cyclase NicD-based signalling mechanism of nutrient-induced dispersion by Pseudomonas aeruginosa. Mol. Microbiol (2014). doi: 10.1111/mmi.12802.

22. Landry RM, An D, Hupp JT, Singh PK, Parsek MR. Mucin-Pseudomonas aeruginosa interactions promote biofilm formation and antibiotic resistance. Mol. Microbiol 59, 142-151 (2006). [PubMed: 16359324]

23. Secor PR, Michaels LA, Ratjen A, Jennings LK, Singh PK. Entropically driven aggregation of bacteria by host polymers promotes antibiotic tolerance in Pseudomonas aeruginosa. Proc. Natl. Acad. Sci (2018).

24. Cattoir V, Narasimhan G, Skurnik D, Aschard H, Roux D, Ramphal R, Jyot J, Lory S. Transcriptional response of mucoid Pseudomonas aeruginosa to human respiratory mucus. MBio. 3, 1-10 (2012).

25. Duan K, Surette MG. Environmental regulation of Pseudomonas aeruginosa PAO1 las and Rhl quorum-sensing systems. J. Bacteriol 189, 4827-4836 (2007). [PubMed: 17449617]

26. Arora SK, Ritchings BW, Almira EC, Lory S, Ramphal R. The Pseudomonas aeruginosa flagellar cap protein, FliD, is responsible for mucin adhesion. Infect. Immun 66, 1000-1007 (1998). [PubMed: 9488388]

27. Chua SL, Hultqvist LD, Yuan M, Rybtke M, Nielsen TE, Givskov M, Tolker-Nielsen T, and Yang L. In vitro and in vivo generation and characterization of Pseudomonas aeruginosa biofilmdispersed cells via c-di-GMP manipulation. Nat. Protoc 10, 1165-1180 (2015). [PubMed: 26158442]

28. Frenkel ES, Ribbeck K. Salivary mucins protect surfaces from colonization by cariogenic bacteria. Appl. Environ. Microbiol 81, 332-338 (2015). [PubMed: 25344244]

29. Kavanaugh NL, Zhang AQ, Nobile CJ, Johnson AD, Ribbeck K. Mucins suppress virulence traits of Candida albicans. MBio. 5, 1-8 (2014).

30. Caldara M, Friedlander RS, Kavanaugh NL, Aizenberg J, Foster KR, Ribbeck K. Mucin biopolymers prevent bacterial aggregation by retaining cells in the free-swimming state. Curr. Biol 22, 2325-2330 (2012). [PubMed: 23142047]

31. Lieleg O, Lieleg C, Bloom J, Buck CB, Ribbeck K. Mucin biopolymers as broad-spectrum antiviral agents. Biomacromolecules. 13, 1724-1732 (2012). [PubMed: 22475261]

32. Huang Y, Mechref Y, Novotny MV. Microscale nonreductive release of O-linked glycans for subsequent analysis through MALDI mass spectrometry and capillary electrophoresis. Anal. Chem 73, 6063-6069 (2001). [PubMed: 11791581]

33. Packer NH, Lawson MA, Jardine DR, Redmond JW. A general approach to desalting oligosaccharides released from glycoproteins. Glycoconj. J 15, 737-747 (1998). [PubMed: 9870349]

34. Chen F-TA, Evangelista RA. Profiling glycoprotein N-linked oligosaccharide by capillary electrophoresis. Electrophoresis. 19, 2639-2644 (1998). [PubMed: 9848672]

35. Guttman A. Analysis of monosaccharide composition by capillary electrophoresis. J. Chromatogr. A 763, 271-277 (1997). [PubMed: 9129325]

36. Taniguchi T, Woodward AM, Magnelli P, McColgan NM, Lehoux S, Jacobo SMP, Mauris J, Argüeso P. N-Glycosylation affects the stability and barrier function of the MUC16 mucin. J. Biol. Chem 292, 11079-11090 (2017). [PubMed: 28487369]

37. Afgan E, Baker D, van den Beek M, Blankenberg D, Bouvier D, Čech M, Chilton J, Clements D, Coraor N, Eberhard C, Grüning B, Guerler A, Hillman-Jackson J, Von Kuster G, Rasche E, Soranzo N, Turaga N, Taylor J, Nekrutenko A, Goecks J. The Galaxy platform for accessible, reproducible and collaborative biomedical analyses: 2018 update. Nucleic Acids Res. 46, W537W544 (2018). [PubMed: 29790989]

38. Love MI, Huber W, Anders S. Moderated estimation of fold change and dispersion for RNA-seq data with DESeq2. Genome Biol. 15 (2014), doi:10.1186/s13059-014-0550-8. 
39. Caballero A, Thibodeaux B, Marquart M, Traidej M, O'Callaghan R. Pseudomonas Keratitis: Protease IV Gene Conservation, Distribution, and Production Relative to Virulence and Other Pseudomonas Proteases. Investig. Ophthalmol. Vis. Sci 45, 522-530 (2004).

40. Howe TR, Iglewski BH. Isolation and characterization of alkaline protease-deficient mutants of Pseudomonas aeruginosa in vitro and in a mouse eye model. Infect. Immun 43, 1058-1063 (1984). [PubMed: 6421735]

41. Dumas Z, Ross-Gillespie A, Kümmerli R. Switching between apparently redundant iron-uptake mechanisms benefits bacteria in changeable environments. Proc. R. Soc. B Biol. Sci 280 (2013), doi:10.1098/rspb.2013.1055.

42. Junker LM, Clardy J. High-throughput screens for small-molecule inhibitors of Pseudomonas aeruginosa biofilm development. Antimicrob. Agents Chemother 51, 3582-3590 (2007). [PubMed: 17664319]

43. Roy S, Elgharably H, Sinha M, Ganesh K, Chaney S, Mann E, Miller C, Khanna S, Bergdall VK, Powell HM, Cook CH, Gordillo GM, Wozniak DJ, Sen CK. Mixed-species biofilm compromises wound healing by disrupting epidermal barrier function. J. Pathol 233, 331-343 (2014). [PubMed: 24771509]

44. Borlee BR, Goldman AD, Murakami K, Samudrala R, Wozniak DJ, Parsek MR. Pseudomonas aeruginosa uses a cyclic-di-GMP-regulated adhesin to reinforce the biofilm extracellular matrix. Mol. Microbiol 75: 827-842 (2010). [PubMed: 20088866] 
a.

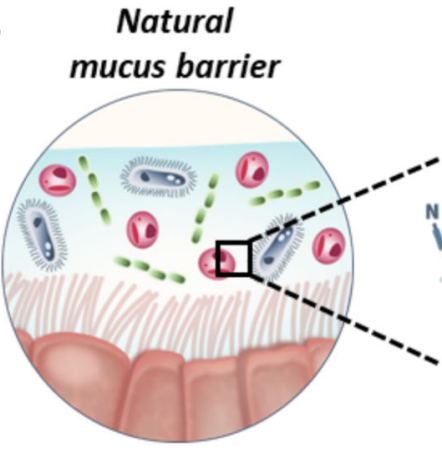

c.

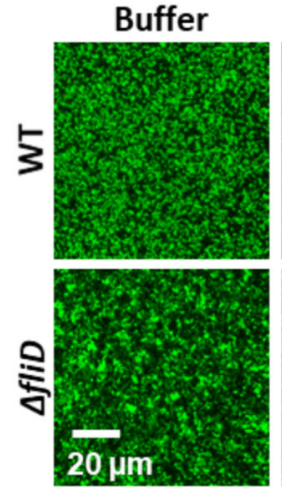

Mucus

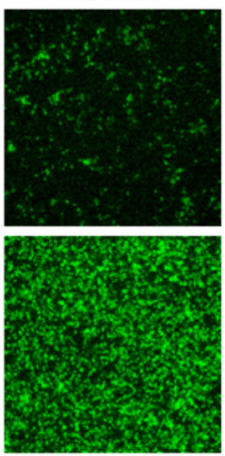

Gel-forming mucins

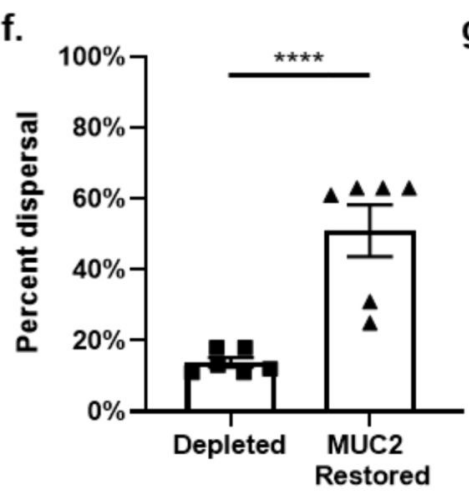

g.

d.

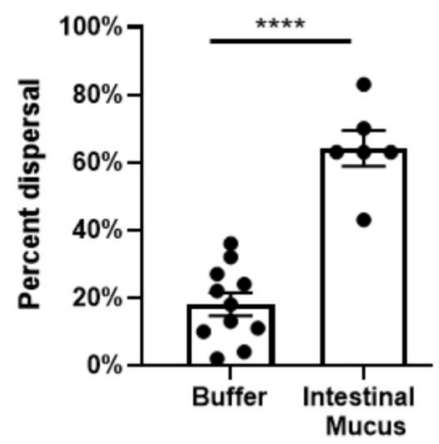

Mucin-glycans

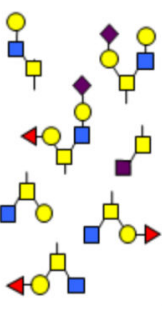

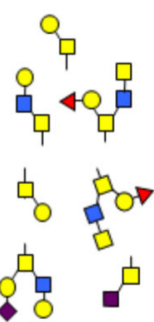

b.

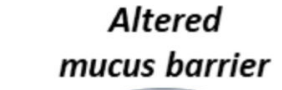

- - Intestinal mucus (baseline)

e.
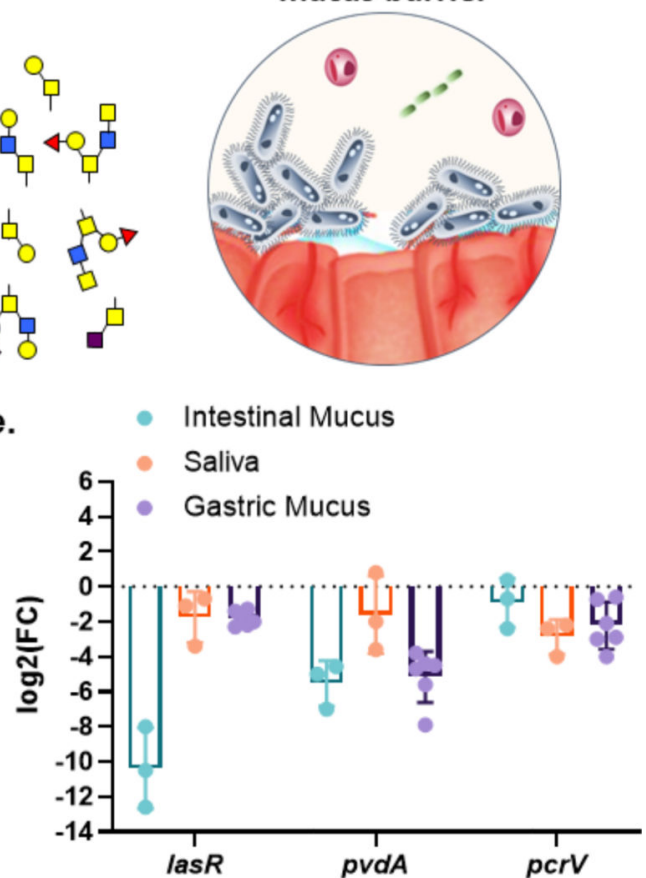

- MUC2 depleted

- - Gastric mucus (baseline)

h.

- MUC5AC depleted
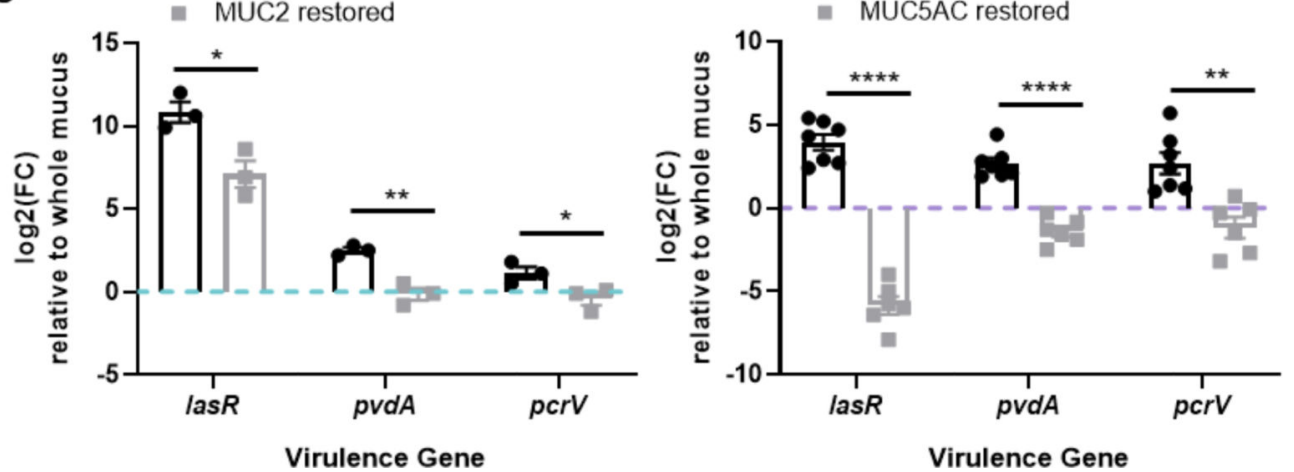

Fig. 1. Native whole mucus suppresses virulence traits in the opportunistic pathogen $P$. aeruginosa.

(a) The natural mucus barrier hosts a diversity of microbes, while limiting infections at the mucosa. Mucins are the major structural component of mucus and are densely grafted with complex glycans. (b) Defects in mucus production are associated with disease and biofilm formation. (c) Representative images of GFP-expressing $P$. aeruginosa biofilms after 3-h treatment with buffer or native mucus reveal that native intestinal mucus reduces biofilm biomass in the wild-type (WT) strain, but not in the flagellar mutant $(\Delta f l i D)$. Similar results were observed in different fields of view across three independent replicates. (d) Native intestinal mucus solutions disperse biofilm biomass into the planktonic state. Percent dispersal is based on the ratio of planktonic cells to total biomass (planktonic cells + remaining biofilm cells). Data and are from biologically independent replicates: Buffer ( $n=$ $12)$, intestinal mucus $(n=6)$. Measure of center is mean \pm standard error. Significance tested 
with two-sided student's $t$ test, $* * * * p<0.0001$. (e) Native mucus solutions suppress key virulence traits relative to mucus solubilization buffer. (f) Depletion of intestinal mucus components $\geq 100 \mathrm{kDa}$ prevents biofilm dispersal. Supplementation of mucus filtrates with exogenous purified MUC2 partially restores biofilm dispersal. Measure of center is mean \pm standard error, and is calculated from 6 biologically independent replicates. Significance tested with two-sided student's $t$ test, **** $p<0.0001$. (g) Depletion of intestinal mucus components $>100 \mathrm{kDa}$ results in increased expression of virulence genes. Supplementation of mucus filtrates with exogenous purified MUC2 partially restores downregulation of virulence genes. (h) Depletion of gastric mucus components $\geq 100 \mathrm{kDa}$ increases the expression of virulence genes. Supplementation of mucus filtrates with exogenous purified MUC5AC restores downregulation of virulence genes. (e, g, h) Data are $\log 2$-transformed qPCR measurements of relative gene expression (fold change, FC). Measure of center is mean \pm standard error, and are calculated from biologically independent replicates: gastric mucus $(n=6)$, intestinal mucus $(n=3)$, saliva $(n=3)$. $(\mathrm{g}, \mathrm{h})$ Significance assessed with twosided student's $t$ test, followed by multiple comparison correction using the Holm-Sidak method, $* p<0.05, * * p<0.01, * * * p<0.001, * * * * p<0.0001$ 
a.
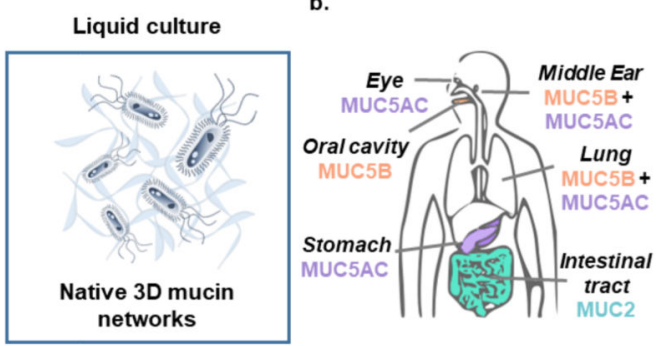

e.

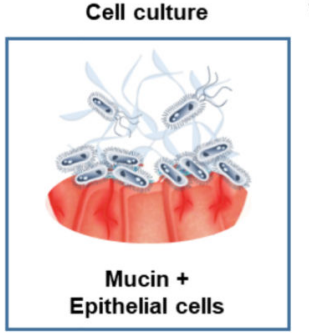

f.

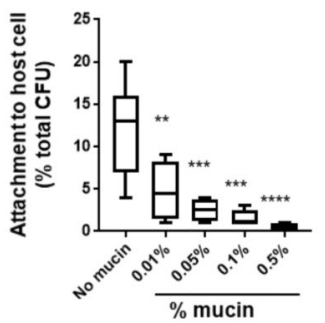

i.

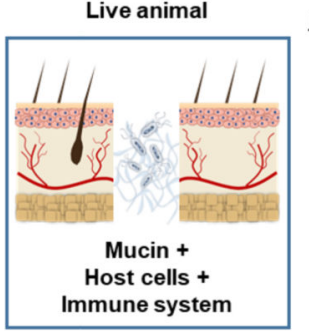

j.

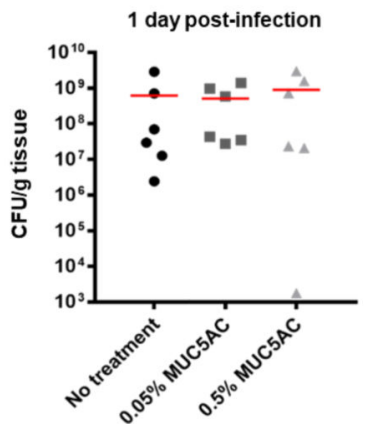

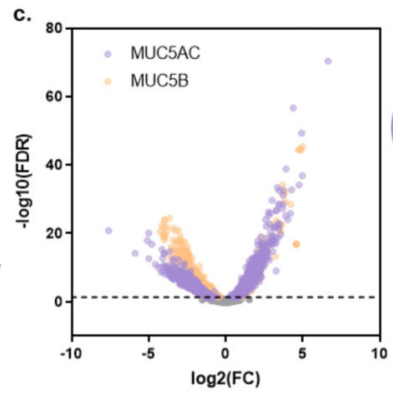

g.
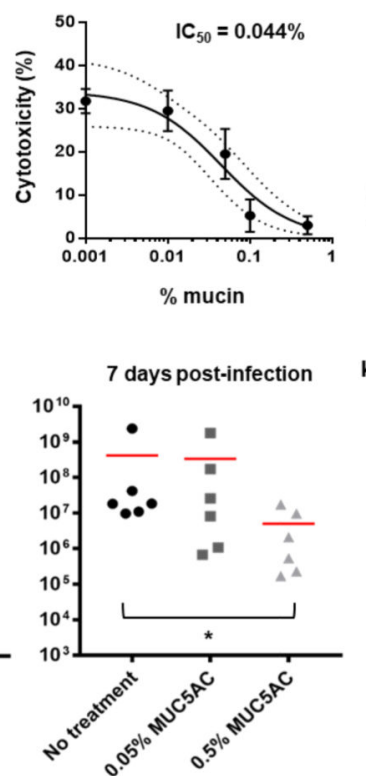

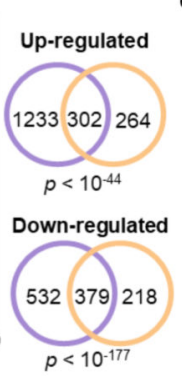

h.
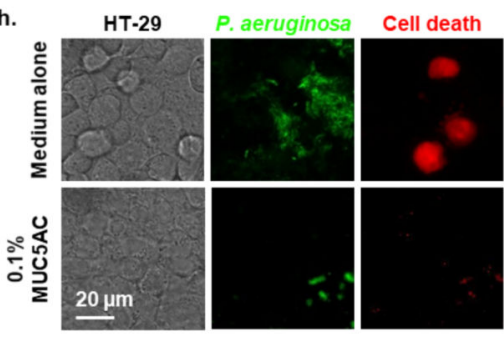

Merge

Fig. 2. Mucins are sufficient to attenuate $P$. aeruginosa virulence in vitro and in vivo.

(a) Gene expression was evaluated in liquid culture with or without the native mucin network. (b) The predominant gel-forming mucins secreted into mucosal niches throughout the body. Sources of mucin used in this study are highlighted. (c) MUC5AC and MUC5B elicit global transcriptional responses in $P$. aeruginosa PAO1. A complete list of fold-changes (FC) and false discovery rates (FDR) appear in Table S1. Fold-change data are average measurements. FDR was determined using the Benjamini Hochberg $p$-value adjustment method. Data are biologically independent replicates: no mucin treatment $(n=6)$,

MUC5AC-treated $(n=3)$, MUC5B-treated $(n=3)$. Correspondence plots of the FC values are presented in Figure S2. Principal component analysis of expression data appears in Figure S13. Venn diagrams contain the total number of genes differentially expressed (FDR $<0.05$ ) after exposure to $0.5 \% \mathrm{w} / \mathrm{v}$ MUC5AC (purple) or MUC5B (orange). Significance of overlap was tested using the hypergeometric test. Functional enrichment analysis of the nonoverlapping regions of the Venn diagrams appears in Figure S2. (d) Functional enrichment analyses identify key virulence pathways among downregulated genes. Significance of enrichment was assessed using the one-sided Mann-Whitney $U$ test, where ranking was based on mean $\log 2$-transformed fold changes from biologically independent replicates: no mucin treatment $(n=6)$, MUC5AC-treated $(n=3)$, MUC5B-treated $(n=3)$. Bars, FDR. Red line, $\mathrm{FDR}=0.05$. (e) $P$. aeruginosa pathogenicity was evaluated in cell culture (containing a 
single human epithelial cell type, HT-29). (f) Exposure to increasing MUC5AC concentrations inhibits $P$. aeruginosa attachment to HT-29 cells. CFU, colony-forming units. Center line, median; box limits, upper and lower quartiles; whiskers, $1.5 \mathrm{x}$ interquartile range. Data are biologically independent replicates: no mucin $(n=7), 0.01 \%$ mucin $(n=4)$, $0.05 \%$ mucin $(n=4), 0.1 \%$ mucin $(n=4), 0.5 \%$ mucin $(n=7)$. Significance was tested in relation to the medium-alone control by ordinary one-way ANOVA, followed by Dunnett's multiple comparisons test, $* * p<0.01, * * * p<0.001, * * * * p<0.0001$. (g) MUC5AC protects HT-29 epithelial cells from death in a concentration-dependent manner. Dotted lines indicate the $95 \%$ confidence interval for the dose-response curve. Data are based on bulk measurements of propidium iodide fluorescence $7.5 \mathrm{~h}$ after infection. Data are mean \pm standard error, $n=4$ biologically independent replicates. (h) MUC5AC maintains the intact epithelial cell monolayer and prevents the onset of HT-29 cellular rounding, bacterial attachment, and HT-29 death. Representative confocal microscopy of HT-29 epithelial cells (bright field), GFP-expressing $P$. aeruginosa PAO1 cells (green), and propidium iodide staining (red) after exposing HT-29 cells to $P$. aeruginosa for $5 \mathrm{~h}$ (top) to $6 \mathrm{~h}$ (bottom) with MUC5AC as indicated. Similar results were observed in different fields of view across three independent replicates. (i) Bacterial viability was monitored in a live dermal wound model (containing living tissue, immune cells, and secreted factors). (j) Bacterial burden on porcine burn wounds decreases after treatment with $0.5 \%$ MUC5AC for 7 days. Symbols represent $P$. aeruginosa $\mathrm{PAO} 1$ burden on 6 individual biopsies collected from two pigs following no treatment (circle), treatment with $0.05 \%$ MUC5AC (square), or treatment with $0.5 \%$ MUC5AC (triangle). Center bar indicates the mean bacterial burden. Significance tested with the Kruskal-Wallis test, followed by Dunn's multiple comparisons test, ${ }^{*} p<0.05$. (k) MUC5AC in isolation does not alter $P$. aeruginosa viability relative to medium alone. Data are mean CFU, colony-forming units, \pm standard error, $n=3$ biologically independent replicates. 


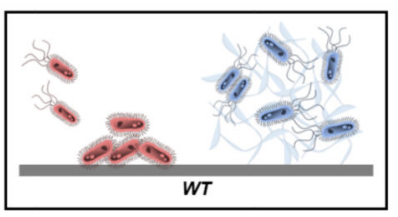

b.
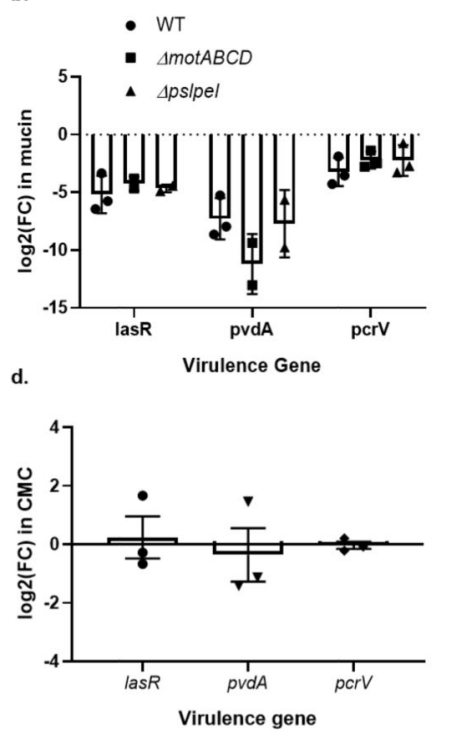
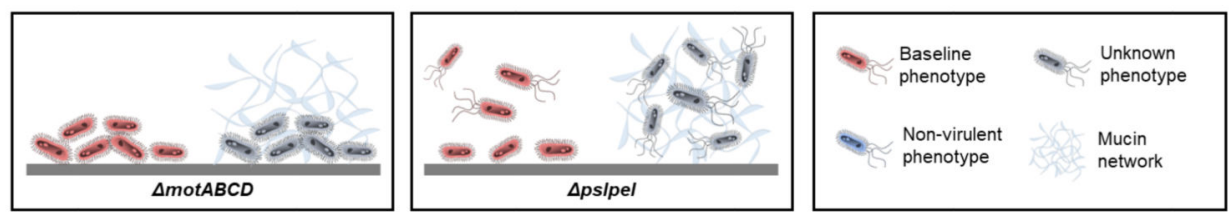

\begin{tabular}{|l|l|c|c|c|c|}
\hline $\begin{array}{l}\text { Regulatory } \\
\text { Cascade }\end{array}$ & Quorum sensing & Siderophore & c-di-GMP & cAMP/vfr & Gac/Rsm \\
\hline $\begin{array}{l}\text { Regulatory } \\
\text { components }\end{array}$ & LasR, RhIR, MvfR & Fur, PvdS, PchR & $\begin{array}{l}\text { SiaA, SadC, RoeA, } \\
\text { WspR }\end{array}$ & CyaA/B, Vfr, PilA & GacS, RsmA \\
\hline $\begin{array}{l}\text { Downstream } \\
\text { Regulon }\end{array}$ & $\begin{array}{l}\text { Phenazine } \\
\text { Type 1 Secretion } \\
\text { Type 2 Secretion } \\
\text { Biofilm formation }\end{array}$ & $\begin{array}{l}\text { Pyoverdine } \\
\text { Pyochelin }\end{array}$ & $\begin{array}{l}\text { Biofilm formation } \\
\text { Biofilm dispersal } \\
\text { Siderophores }\end{array}$ & $\begin{array}{l}\text { Type 3 Secretion } \\
\text { Motility }\end{array}$ & $\begin{array}{l}\text { Biofilm formation } \\
\text { Type 6 Secretion }\end{array}$ \\
\hline
\end{tabular}

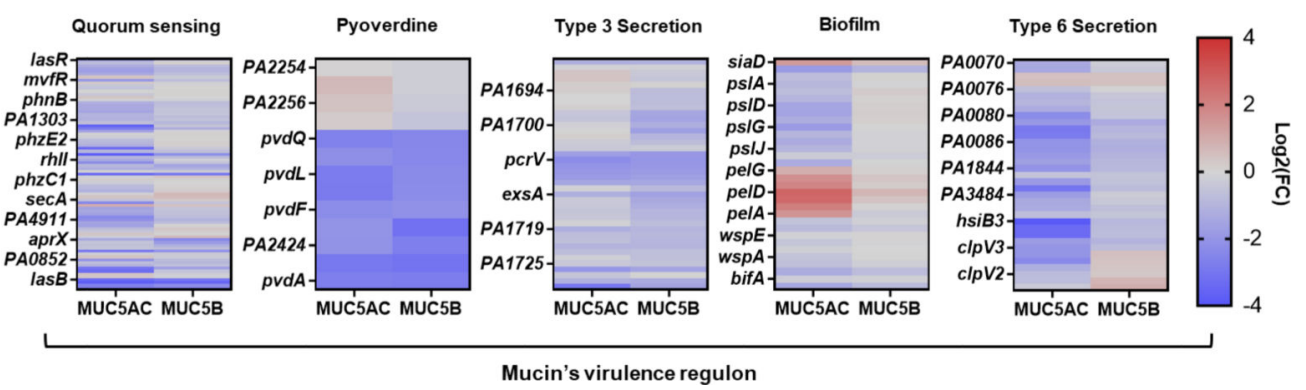

Fig. 3. The virulence systems suppressed by mucin are downstream of multiple, distinct regulatory cascades, and regulation of these systems is independent of bacterial motility and aggregation.

(a) Mucin promotes a motile, non-aggregated phenotype and suppresses virulence in $P$. aeruginosa PAO1. To determine whether changes to virulence are caused by a shift in motility or aggregation, we monitored the virulence phenotype of non-motile ( $\triangle$ motABCD) and non-aggregative ( $\triangle$ pslpel) mutants after mucin exposure. (b) Downregulation of virulence gene expression by mucin does not require a shift in motility or aggregation, indicating that it is a parallel effect of mucin. Data are log2-transformed qPCR measurements of relative gene expression \pm standard error, $n=3$ biologically independent replicates. Significance was assessed by multiple two-tailed $t$ test, followed by Holm-Sidak correction for multiple comparisons, no significant difference in log2(fold change) between the WT and mutants. (c) Mucin's virulence regulon is downstream of multiple, interconnected regulatory cascades. (d) Carboxymethylcellulose (CMC) does not differentially regulate virulence genes. Data are $\log 2$-transformed qPCR measurements of relative gene expression \pm standard error, $n=3$ biologically independent replicates.

Significance was assessed by one sample two-tailed $t$ test, followed by Bonferroni correction for multiple comparisons, no significant difference in $\log 2$ (fold change) between CMC and medium alone. 
a.

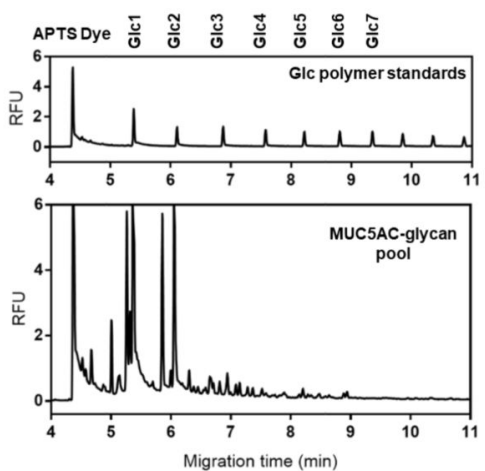

D.

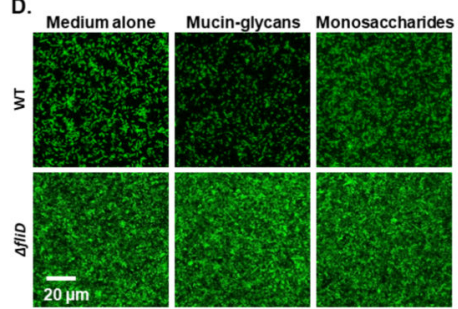

i. Downregulated virulence pathways

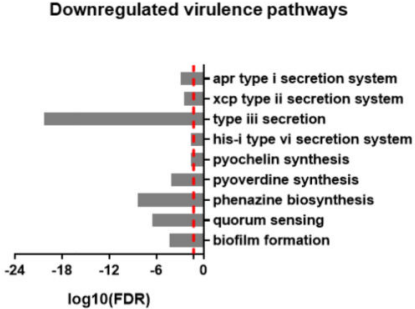

b.
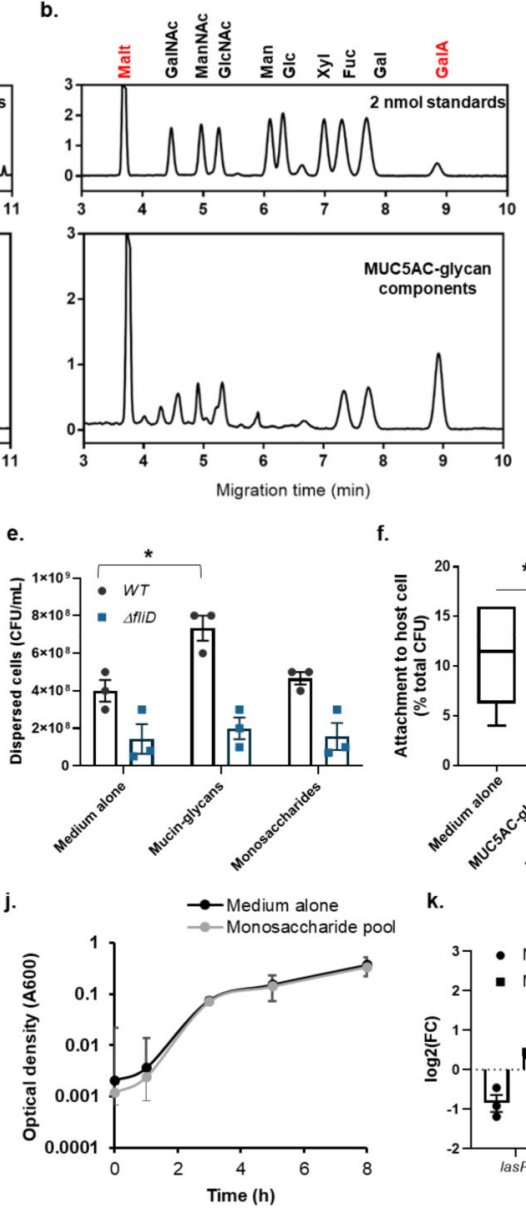
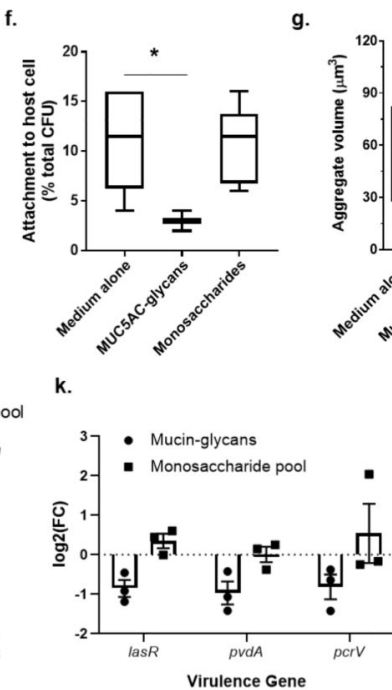

g.
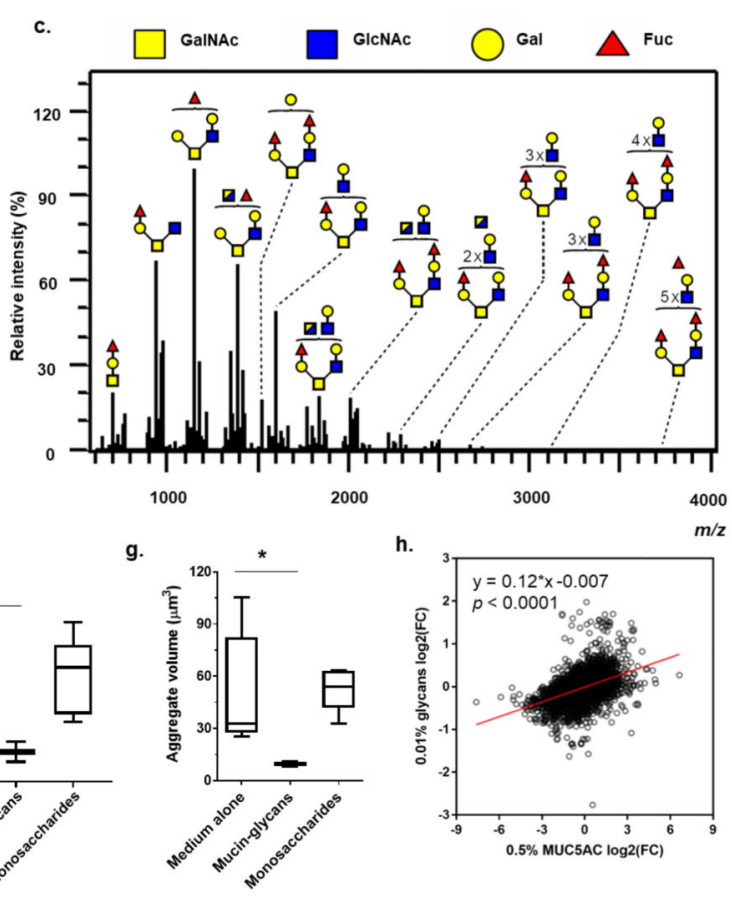

h.

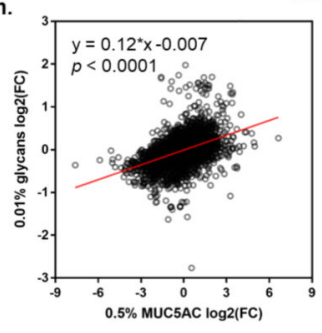

Fig. 4. Complex $\boldsymbol{O}$-glycans are the major regulatory component of MUC5AC.

(a) Oligosaccharides released by alkaline $\beta$-elimination were resolved via capillary electrophoresis. The mucin-glycan pool includes extended chains consisting of $>7$ residues.

(b) Monosaccharide composition of mucin-glycans were assessed via capillary electrophoresis. These mucin oligosaccharides are predominantly $O$-linked, as evidenced by the Man ( $N$-linked) to GalNAc ( $O$-linked) ratio. Red labels indicate the quantitation standard maltose (Malt) and the migration standard galacturonic acid (GalA). Abbreviations and migration times (in minutes) for monosaccharide standards: $\mathrm{N}$-acetylgalactosamine (GalNAc) 4.5, N-acetylmannosamine (ManNAc) from $\mathrm{N}$-acetylneuraminic acid 4.95, Nacetylglucosamine (GlcNAc) 5.26, mannose (Man) 6.08, glucose (Glc) 6.33, xylose (Xyl) 6.99 , fucose (Fuc) 7.29, galactose (Gal) 7.67. (c) MALDI-TOF spectrum of $O$-glycans from MUC5AC. $m / z$, mass/charge values. The complete list of $O$-glycan structures with experimental and theoretical masses appears in Table S8. (a-c) Similar results have been observed in 3 independent replicates. (d) Representative images of GFP-expressing $P$. aeruginosa biofilms after a 3 -h treatment with medium alone, with $0.01 \%$ mucin-glycans, or with a $0.01 \%$ pool of monosaccharides. Glycan solutions reduce biofilm biomass in the wild-type (WT) strain, but not the flagellar mutant $(\Delta f l i D)$. Similar results were observed in different fields of view across three independent replicates. (e) Mucin-glycans, but not 
monosaccharides, disperse biofilm biomass into the planktonic state for the wild-type (WT) strain, but not the flagellar mutant $(\Delta f l i D)$. Center line, mean \pm standard error, $n=3$ biologically independent replicates. Significance was tested in relation to the medium-alone control by ordinary one-way ANOVA, followed by Dunnett's multiple comparisons test, $* p<0.05$ (f) Mucin-glycans inhibit bacterial attachment to human epithelial HT-29 cells. Center line, median; box limits, upper and lower quartiles; whiskers, $1.5 \mathrm{x}$ interquartile range. Data are biologically independent replicates: medium alone $(n=6)$, MUC5ACglycans $(n=3)$, monosaccharides $(n=6)$. Significance was tested in relation to the mediumalone control by ordinary one-way ANOVA, followed by Dunnett's multiple comparisons test, ${ }^{*} p<0.05$. (g) Relative size distributions of aggregates identified via live 3D confocal microscopy and analyzed by COMSTAT. In medium alone and medium with monosaccharides, $P$. aeruginosa biomass is concentrated in large surface-associated aggregates, whereas MUC5AC-glycans suppress the formation of aggregates. Center line, median; box limits, upper and lower quartiles; whiskers, $1.5 \mathrm{x}$ interquartile range. Data are average aggregate sizes compiled from six separate z-stacks. Significance tested with the Kruskal-Wallis test, followed by Dunn's multiple comparisons test, ${ }^{*} p<0.05$. (h) Low concentrations of MUC5AC-glycans elicit a transcriptional response that positively correlates with transcriptional changes elicited by whole MUC5AC. Fold-change data are average measurements from 3 biologically independent replicates. Significance tested with the regression slope test. (i) Free glycans suppress the same virulence pathways as whole mucin. Significance of enrichment was assessed using the Mann-Whitney U test, where ranking was based on mean log2-transformed fold changes from 3 biologically independent replicates. (j) Growth is not altered by the presence of the monosaccharide components in mucin-glycans. Data are mean $\mathrm{OD}_{600} \pm$ standard error, $n=3$ biologically independent replicates. (k) Complex mucin-glycans, but not their monosaccharide components, induce expression changes in signature virulence genes. Data are qPCR measurements of relative gene expression \pm standard error, $n=3$ biologically independent replicates. (l) Bacterial burden on porcine burn wounds decreases after treatment with $0.1 \%$ mucin-glycans for 7 days. Symbols represent $P$. aeruginosa PAO1 burden on individual biopsies collected burns following no treatment (circle, $n=6$ ), treatment with $0.1 \%$ MUC5AC-glycans (square, $n=$ 3 ), or treatment with $0.1 \%$ monosaccharides (triangle, $n=3$ ). Center bar indicates the mean bacterial burden. Significance tested in relation to the no-treatment control with the KruskalWallis test, followed by Dunn's multiple comparisons test, ${ }^{*} p<0.05$. 